\title{
EXOPLANETS
}

\section{How to lose an atmosphere}

Astrophys. J. (in the press); preprint at

https://arxiv.org/abs/1912.08820

Planetary atmospheres lose material with time. This process is particularly effective during the early stages of their evolution. Indeed, terrestrial planets retain little or none of their primordial atmospheres, which are mostly made up of hydrogen and helium. Photoionization of atmospheric molecules, due to the intense X-ray and extreme UV radiation from the young star, and loss by impacts - which are more frequent in the early days of planetary systems - are considered to be the main causes for this erosion. Alex Howe and colleagues show instead that photodissociation driven by less energetic UV photons (here between 121 and $275 \mathrm{~nm}$ ) can be the dominant source of atmospheric mass loss.

Howe et al. compute the mass loss of the atmosphere for an Earth-like planet at Earth-Sun distance by different processes: Jeans escape, hydrodynamic escape, stellar wind ablation, impact erosion, photoionization, and photodissociation. They assume that the planet formed by pebble accretion, which is a quick process allowing the planet to retain 23,000 bar of gas ( $2 \%$ of its total mass) from the protoplanetary disk before its dissipation. The results show that photodissociation accounts for more than $90 \%$ of the total mass loss, growing more dominant over time. Impacts have a non-negligible effect $(\sim 5 \%)$, while the other four mechanisms are very inefficient. In addition, as photodissociation mostly removes $\mathrm{H}$ and $\mathrm{He}$ molecules, the remaining atmosphere would be enriched in water and other volatiles.

However, only $\sim 5,700$ bar would be removed over the first $500 \mathrm{Myr}$, indicating that some other process must be at play or that initial gas accretion is less efficient than pure pebble accretion models suggest.

Luca Maltagliati

Published online: 3 February 2020

https://doi.org/10.1038/s41550-020-1021-7 